\title{
OBSERVATION OF ANISOTROPY IN THE ARRIVAL DIRECTION DISTRIBUTION OF COSMIC RAYS ABOVE TEV ENERGIES WITH ICECUBE
}

\author{
S.TOSCANO for the ICECUBE Collaboration \\ IceCube Research Center, University of Wisconsin, Madison, WI 53703, U.S.A. \\ E-mail: toscano@icecube.wisc.edu
}

\begin{abstract}
Between May 2009 and May 2010, the IceCube neutrino detector recorded 32 billion of atmospheric muons generated in air showers produced by cosmic rays in the TeV energy range. With such high statistics sample it is possible to observe, for the first time in the southern hemisphere, an energy dependence in the Galactic cosmic ray anisotropy up to a few hundred $\mathrm{TeV}$. This study shows that the same large-scale anisotropy observed at median energies around $20 \mathrm{TeV}$ is not present at $400 \mathrm{TeV}$; the anisotropy observed at $400 \mathrm{TeV}$ shows substantial differences with respect to that at lower energy. In addition to the large-scale features observed at $20 \mathrm{TeV}$ in the form of strong dipole and quadrupole moments, the data include several localized regions of excess and deficit on scales between $10^{\circ}$ to $30^{\circ}$. The features observed at both large and small scale are statistically significant, but their origin is currently unknown.
\end{abstract}

\section{Introduction}

The IceCube detector, deployed around $2000 \mathrm{~m}$ below the surface of the South Polar ice sheet, is designed to detect upward-going neutrinos from astrophysical sources. However, it is also sensitive to downward-going muons produced in cosmic ray air showers. To penetrate the ice and trigger the detector, the muons must possess an energy of at least several hundred $\mathrm{GeV}$, which means they are produced by primary cosmic rays with energies in excess of several TeV. At these energies it is expected that interactions of cosmic rays with Galactic magnetic fields should completely randomize their arrival directions. Nevertheless, at $\mathrm{TeV}$ energies, multiple observations of anisotropy in the arrival direction distribution of cosmic rays have been observed on large and small angular scales by the Tibet $\mathrm{AS}_{\gamma}$ array ${ }^{1}$, Super-Kamiokande ${ }^{2}$, Milagro $3{ }^{34}$, ARGO-YBJ 5]. These measurements were performed in the northern hemisphere. The first observation of the large-scale cosmic ray anisotropy in the southern sky has been reported by the IceCube detector 6 in 2010.

The observations at several $\mathrm{TeV}$ are quite similar across all experiments and between the northern and southern hemispheres. However, at high energies observations from several experiments have been really controversial. For example, the Tibet AS $\gamma$ collaboration has published a null result at $300 \mathrm{TeV}^{1}$, while at $370 \mathrm{TeV}$ the EAS-TOP collaboration has claimed evidence of an anisotropy 7 .

In this proceedings we describe the energy-dependence of the large-scale anisotropy using IceCube data. We also describe a search of the southern sky for anisotropy on small and medium angular scales. 


\section{The IceCube Detector and Data}

IceCube, completed in December 2010, is a $\mathrm{km}^{3}$-size neutrino detector frozen into the glacial ice sheet at the geographic South Pole. High-energy particles passing through the detector emit Cherenkov radiation and their tracks are recorded by an array of 5160 Digital Optical Modules $(\mathrm{DOMs})^{8}$ embedded in the ice. The DOMs are attached to 86 vertical cables, or strings, deployed at depths between $1450 \mathrm{~m}$ and $2450 \mathrm{~m}$ below the surface of the ice sheet. Between 2009 and 2010, 59 strings had already been deployed and the detector was running in this smaller configuration (IC59).

The main trigger used for physics analysis in IceCube is a simple majority trigger which requires coincidence of 8 or more DOMs hit in the deep ice within a $5 \mu s$ window. The trigger rate of downgoing muons in IC59 was $1.7 \mathrm{kHz}$, a factor of $10^{6}$ larger than the neutrino rate. Due to the high rate of muon events, the muon tracks are reconstructed online using a maximum likelihood reconstruction, and the results are stored in a Data Storage and Transfer (DST) format to satisfy bandwidth constraints at the South Pole.

The analysis presented in this paper uses the DST data collected during IC59 physics runs between May 2009 and May 2010. The data set contains approximately $3.4 \times 10^{10}$ muon events detected with an integrated live time of 334.5 days. A cut in zenith angle $\left(\theta>115^{\circ}\right)$ is used to remove misreconstructed tracks near the horizon, reducing the final data set to $3.2 \times 10^{10}$ downgoing events. Simulations indicate that the median angular resolution is $3^{\circ}$ and the median primary energy of the cosmic ray data set is $20 \mathrm{TeV}$. The primary cosmic ray energy resolution is of order 0.5 in $\Delta \log (E)$.

\section{Data Analysis and Results}

\subsection{Evolution of the Anisotropy with Energy}

Because we must measure cosmic rays indirectly via observations of muons produced in the extensive air showers, the energy of the cosmic ray primary particles is inferred using estimates of the muon energy.

In this analysis the estimate of cosmic ray energy is based on the number of DOMs hit by Cherenkov photons (i.e. number of channels, or $N_{c h}$ ). Due to the zenith angle $(\theta)$ dependence of the relation between $N_{c h}$ and the cosmic ray primary energy, a two dimensional cut in $N_{c h}$ and $\theta$ is used to divide the data into two energy samples. The low energy sample contains events with a median energy of $20 \mathrm{TeV}$, where $68 \%$ of the events are between 4 and $63 \mathrm{TeV}$; and the high energy sample contains events with a median energy of $400 \mathrm{TeV}$, with $68 \%$ of the events between 100 and $1258 \mathrm{TeV}$.

We apply the same method used in the past analysis $[6$ to investigate the arrival direction distribution of the cosmic rays at these two energies. Equatorial maps of relative intensity are produced for the two energy samples and shown in Fig. 1, The sky is binned into an equal-area grid with a resolution of $0.9^{\circ}$ using the publicly-available HEALPix ${ }^{9}$ library, and smoothed with $3^{\circ}$ to match the data angular resolution of the online muon reconstruction.

To study the evolution of the anisotropy with energy, we project the maps in right ascension and fit the resulting 1D distribution to a first and second-order harmonic function of the form: $\sum_{j=1}^{2}=A_{j} \cos \left[j\left(\alpha-\phi_{j}\right)\right]+B$, where $\left(A_{j}, \phi_{j}\right)$ are the amplitude and phase of the anisotropy, $\alpha$ is the right ascension, and $\mathrm{B}$ is a constant. Fig. 2 shows the right ascension projection of the maps from Fig. 1 for the two energy samples together with the fit result. The error bars represent the statistical errors.

The anisotropy observed at $20 \mathrm{TeV}$ with IC59 is consistent with the previously reported observation with IceCube-22 6], confirming the continuation of the arrival distribution pattern observed in the northern hemisphere to the southern sky. At $400 \mathrm{TeV}$ the sky map and the 

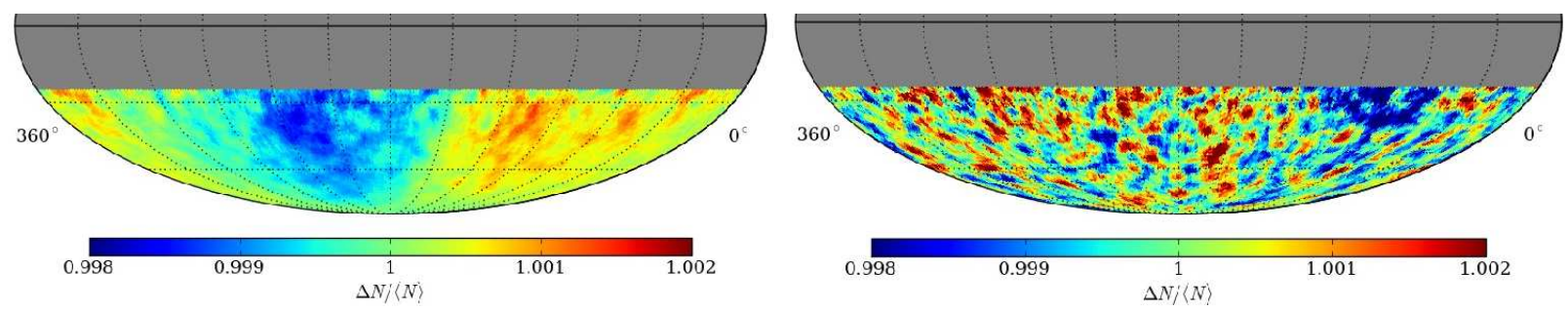

Figure 1: Relative intensity $\Delta N /\langle N\rangle$ of the IC59 data in equatorial coordinates for the $20 \mathrm{TeV}$ (left) and the 400 $\mathrm{TeV}$ (right) energy sample. The sky maps are smoothed within $3^{\circ}$ to match the angular resolution of the data.

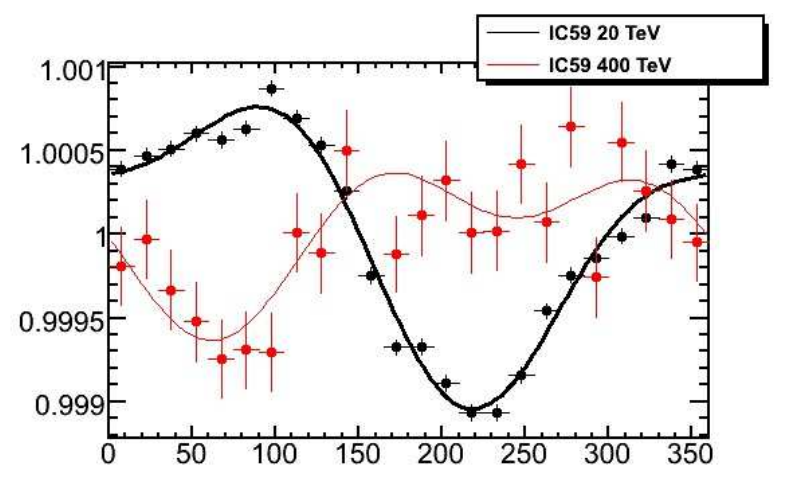

Figure 2: One-dimensional projection in right ascension $\alpha$ of the $20 \mathrm{TeV}$ (black) and $400 \mathrm{TeV}$ (red) maps in Fig. 1 The data are shown with statistical uncertainties, and the lines correspond to the first and second harmonic fit.

right ascension projection show substantial differences with respect to the observation at lower energy. The large scale structure visible at $20 \mathrm{TeV}$ vanishes and a smaller (of $\sim 20^{\circ}$ extension) deficit appears at $\left(\alpha=73.1^{\circ}, \delta=-25.3^{\circ}\right)$ with a post-trials significance of $-6.3 \sigma$. A significant excess cannot be distinguished yet from the current statistics. This is the first observation of an anisotropy at $400 \mathrm{TeV}$ in the southern sky 10 .

\subsection{Medium-Small Scale Structures}

The arrival direction distribution of cosmic rays observed in IC59 is dominated by the presence of large structures in the form of a strong dipole and quadrupole component. In order to search for correlations on smaller angular scales a complementary method has been used to analyse the data. The first step in this search is the creation of a "reference map" to which the actual data map is compared. The reference map essentially shows what the sky map would look like if the cosmic ray flux was isotropic. The reference map accounts for detector effects that might cause a spurious anisotropy, such as non-uniform exposure to different parts of the sky or gaps in the uptime, and it is created using a background estimation method based on the time scrambling technique described in Alexandreas et al. 11

The complete analysis, reported in $\mathrm{R}$. Abbasi et al ${ }^{12}$, can be summarised as follows:

- The muon events are binned in equatorial coordinates to create a $2 \mathrm{D}$ map of event counts $N_{i}(\alpha, \delta)$. (Note that $i$ is the pixel index in the binned map.) The reference counts $\left\langle N_{i}(\alpha, \delta)\right\rangle$ are estimated from the data, and deviations from isotropy are calculated in each bin. The deviations can be expressed in terms of a relative intensity $\delta I_{i}=\left(N_{i}-\left\langle N_{i}\right\rangle\right) /\left\langle N_{i}\right\rangle$, or in terms of statistical significance using the method of Li and $\mathrm{Ma}^{13}$.

- The angular power spectrum of the relative intensity map is calculated to determine correlations between pixels at several angular scales. The angular power spectrum of the IC59 
data exhibits significant power not only at the largest angular scales, but also down to scales of about $10^{\circ}$.

- A fit of the dipole and quadrupole terms to the relative intensity map is performed and subtracted from the intensity map in order to obtain a map of residual counts, which is then analyzed for small-scale structure.

- To increase the sensitivity to the small-scale structures in the data, we apply a smoothing procedure which takes the reference level and residual data counts in each bin and adds the counts from pixels within some angular radius of the bin.
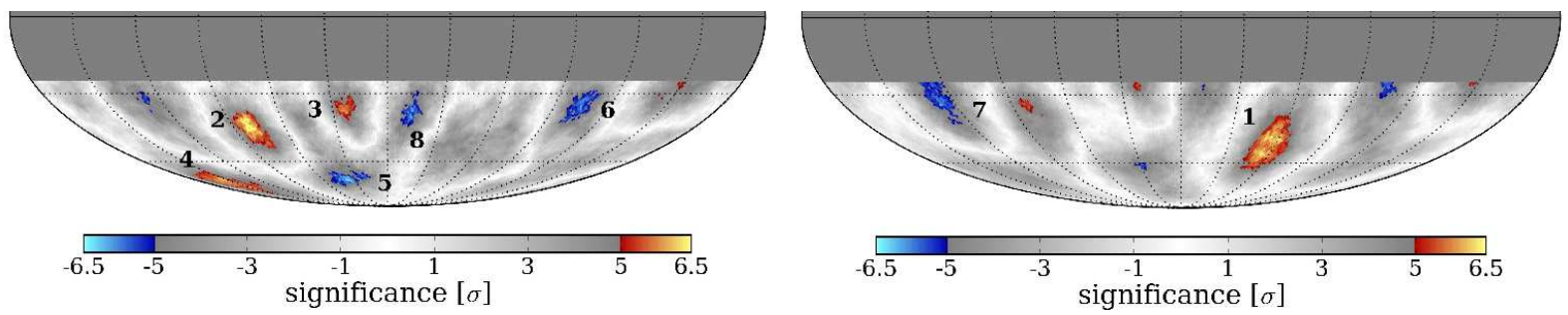

Figure 3: Left: Significances of the IC59 residual map plotted with $12^{\circ}$ smoothing. Right: Significances of the IC59 residual map plotted with $20^{\circ}$ smoothing. The regions with a pre-trial significance larger than $\pm 5 \sigma$ are shown in colours.

Fig. 3 shows the smoothed residual maps of significance in equatorial coordinates. No energy selection has been done for this analysis; the maps show the sky at a median energy of $20 \mathrm{TeV}$ (Sec. 2). We identify eight regions, shown in colours, with a pre-trial significance larger than $\pm 5 \sigma$. Since the optimal scales vary from region to region and no single smoothing scale shows all regions, we show the maps with two smoothing scales, $12^{\circ}$ (left) and $20^{\circ}$ (right). The most significant excess on the sky is Region 1 at $\left(\alpha=122.4^{\circ}, \delta=-47.4^{\circ}\right)$, with a peak significance of $7.0 \sigma$ at a smoothing radius of $22^{\circ}$ which is reduced to $5.3 \sigma$ after accounting for trials.

\section{Conclusions}

Using $3.2 \times 10^{10}$ events recorded with the partially deployed IceCube detector between May 2009 and May 2010, we have found that the arrival direction distribution of cosmic rays at several $\mathrm{TeV}$ exhibits significant anisotropy on several angular scales. The data are dominated by dipole and quadrupole moments, but there is also significant structure on angular scales down to about $10^{\circ}$. At $400 \mathrm{TeV}$ the large structure seems to vanish and the most significant region is a relative deficit at a right ascension where the broad excess dominated at $20 \mathrm{TeV}$.

\section{References}

1. M. Amenomori et al, Astrophys. J. 626, L29 (2005).

2. G. Guillian et al, Phys. Rev. D 75, 062003 (2007).

3. A.A. Abdo et al, Phys. Rev. Lett. 101, 221101 (2008).

4. A.A. Abdo et al, Astrophys. J. 698, 2121 (2009).

5. S. Vernetto et al, Proc. 31st ICRC, Łódź, Poland (2009).

6. R.Abbasi et al, Astrophys. J. 718, L194 (2010).

7. M. Aglietta et al, Astrophys. J. 692, L130 (2009).

8. R. Abbasi et al, Nucl. Instrum. Methods A 618, 139 (2010).

9. K.M. Gorski et al, Astrophys. J. 622, 759 (2005).

10. R. Abbasi et al, submitted to Astrophys. J, arXiv:1109.1017 (2011).

11. D.E. Alexandreas et al, Nucl. Instrum. Methods A 328, 570 (1993).

12. R. Abbasi et al, Astrophys. J. 740, 16 (2011).

13. T.-P. Li and Y.-Q.Ma, Astrophys. J. 272, 317 (1983). 